PLCTRS, trial participants will be asked if they can identify which investigational drug was being studied and its possible side effects. This project could anticipate identification of trial elements and results deemed difficult to comprehend by participants; thus they would be better informed after interacting with the platform. DISCUSSION/ SIGNIFICANCE OF FINDINGS: This project will ensure that participants better comprehend the trials they participated in beyond the required informed consent process - which only covers their comprehension prospectively. This project seeks to address the gap of ensuring participant comprehension retrospectively.

57437

Effects of Prebiotics on the Gut Microbiome Profile, Betacell Function and Immune Markers in Newly-Diagnosed Type 1 Diabetes

Heba M. Ismail, Carmella Evans-Molina and Linda A. DiMeglio Indiana University School of Medicine

ABSTRACT IMPACT: The proposed research study will provide critical pilot data on the effect of using the prebiotic (HAMS-AB) on the gut microbiome profile, Beta-cell function and immune markers in humans with T1D. OBJECTIVES/GOALS: The overall objective of this study is to assess how the prebiotic high amylose maize starch that has been acetylated and butyrylated (HAMS-AB) impacts the gut microbiome profile, short chain fatty acid (SCFA) production, glycemia, Beta-cell function/health and immune responses in newly diagnosed youth with type 1 diabetes (T1D). METHODS/STUDY POPULATION: We are performing a pilot randomized cross-over trial. We plan to recruit 12 newly-diagnosed T1D youth with residual Beta-cell function between 12-16 years of age. We will profile the gut microbiome using metagenomics, measure stool SCFA levels using mass spectrometry, assess glycemia using continuous glucose monitoring, assess insulin production using mixed meal tolerance testing, assess Beta-cell stress using proinsulin/C-peptide levels, and test immune responses by examining cytokine levels and frequency, phenotype and function of T cell markers in peripheral blood. RESULTS/ANTICIPATED RESULTS: Thus far, we have enrolled 3 participants, 1 has completed the study. Baseline assessments indicate that we have technical feasibility of performing the above studies and measurements. Recruitment and enrollment are ongoing. We hypothesize that the use of HAMS-AB in newly diagnosed youth with T1D will (i) improve the gut microbiome profile, (ii) increase SCFA production, (iii) improve overall glycemia and Beta-cell function and (iv) modulate the immune system and mitigate autoimmunity. DISCUSSION/SIGNIFICANCE OF FINDINGS: Given the failure to develop a cure for T1D despite multiple completed intervention studies and the unknown long-term effects of immune-modulatory therapy on those at risk for or those diagnosed with T1D, prebiotics such as HAMS$\mathrm{AB}$ may offer a simple, safe, yet inexpensive and tolerated dietary alternative approach to mitigating disease.

64180

The Proportion of Young Patients with Acute Surgical Pain and Development of Opiate Abuse Disorders Armando Uribe-Rivera, Linda Rasubala, Daniela Alvarez, Daniel Monroy-Giamundo, Hans Malmstrom and Yan-Fang Ren University of Rochester Eastman Institute for Oral Health

ABSTRACT IMPACT: The importance of this study is to evaluate the responses following the exposure of opioid drugs in young adults to address the current opioid epidemics OBJECTIVES/GOALS: To compare the proportion of patients between 18 and 25 years of age, who develop an opioid abuse disorder following dental surgery, to those following other surgical procedures, when an opioid drug is used for acute postoperative pain control. METHODS/STUDY POPULATION: We fashioned an IRB-sponsored retrospective cohort study of patients, ages 18 to 25 years old, who presented for dental surgery or other medical surgical procedures, at Strong Memorial Hospital Medical Center, at the University of Rochester and received opioid drug treatments, for acute surgical pain management. Patients with the diagnosis of acute surgical pain were included in the study. However, those with chronic pain or other related abnormalities were excluded, even if a diagnosis of acute surgical pain was present in the electronic chart. The clinical data were retrieved from electronic medical records and NYSiStop records. All statistics were significant at the level of $<0.1$ RESULTS/ANTICIPATED RESULTS: We identified 167 subjects, of whom, only 150 subjects met inclusion criteria $(n=100)$ in dentistry and $(n=50)$ in other medical specialties. Patients were followed up in a 7-year period. Most of the subjects were females $(n=91)$, Caucasian $(n=80)$, and lived in a suburban location $(n=78)$. The most frequently prescribed opioid included hydrocodone $(n=119)$. A significantly higher proportion $(8.7 \%)$ of patients developed opioid abuse disorders in the control group, compared to $1 \%$ of subjects in the experimental

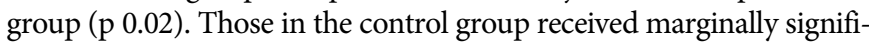
cant higher doses of MMEs 447.2 +/-644.8 $\mathrm{mg}$ vs $306.2+/-354.7 \mathrm{mg}$ in the experimental group ( $\mathrm{p}$ 0.086). Those in the control group had significantly longer periods of opioid treatment $10(+/-12)$ compared to $6(+/-6)$ days in the experimental group DISCUSSION/ SIGNIFICANCE OF FINDINGS: It is paramount to evaluate the morphine milligram equivalents, and duration of opioid treatment given to the young population with acute surgical pain, to prevent opioid abuse disorders in this vulnerable cohort.

70400

\section{Collaborative Care for Opioid Dependence And Pain (CCODAP): A Pilot Randomized Control Trial of an Opioid Tapering Intervention}

Michael Bushey and Kurt Kroenke

Indiana University School of Medicine

ABSTRACT IMPACT: If successful, this program can provide a scalable, patient-centered intervention to help patients taper off opioid medications in primary care settings. OBJECTIVES/GOALS: Tapering of chronic opioid therapy is often desirable but challenging in primary care and specialty clinics that lack behavioral health expertise. The objective of this pilot study is to determine the feasibility of testing a peer-delivered pain self-management program to assist primary care patients through an opioid taper. METHODS/STUDY POPULATION: To provide critical support to patients and providers during opioid medication tapering, we propose to conduct a 40 patient randomized controlled pilot of a 12 week telecare collaborative care program administered by a psychiatrist and peer recovery specialist team. The intervention will incorporate a validated positive psychology intervention for treating chronic pain. Additionally, participants will be invited to participate in semi-structured individual interviews to discuss their experience in the trial, what worked well, what could be improved, and potential strategies to bolster recruitment of additional patients in future studies. RESULTS/ ANTICIPATED RESULTS: Our primary aim is to determine the effectiveness of our intervention in facilitating opioid medication weaning, with reduction in opioid dose as the primary outcome. Our secondary aims will be to assess pain outcomes, adherence to tapering, patient satisfaction, and barriers to adherence as described by patients. 